\title{
Geomagnetic Noise Analysis and Suppression from Next Generation Autonomous Systems 2009 Sea Trials
}

\author{
Michael Hughes \\ SPAWAR Systems Center, Pacific \\ San Diego, California 92152 \\ Email: michael.hughes4@navy.mil
}

\author{
Mihajlo Tomic \\ SPAWAR Systems Center, Pacific \\ San Diego, California 92152 \\ Email:mihajlo.tomic@navy.mil
}

\begin{abstract}
A prominent area of research promoted by naval groups throughout the world involves the advancement of wireless, acoustically-linked, total-field magnetic sensors. In 2009, a Next Generation Autonomous Systems (NGAS) sea trial took place which tested the capabilites of a field of such sensors. During this trial, ambient geomagnetic noise was recorded in an effort to provide an improved understanding of the noise induced on these magnetic sensing systems.

Results from the analysis of the NGAS noise data set are presented in this paper. Four categories of magnetic noise are presented including: acoustic-modem induced noise, clockdrift induced noise, geomagnetic background fluctuations, and geomagnetic ULF pulsations. A robust method for eliminating clock-drift induced noise is presented, which yields over $20 \mathrm{~dB}$ improvement in noise suppression. The statistical and diurnal properties of the geomagnetic background fluctuations are shown. Interestingly, the hourly noise variance was found to reach minimums at around 1 AM UTC each day that were $15 \mathrm{~dB}$ lower than the rest of the day. Additionally, the results of applying two noise-suppression techniques are presented. Finally, the effects of ULF pulsations on a single-sensor detection system are discussed.
\end{abstract}

\section{INTRODUCTION}

An active area of research for naval groups around the world involves the advancement of stationary, sea floor mounted, passive total-field magnetic sensor technologies and associated signal processing algorithms. These technologies are vital to the defense of harbors, ports, and shipping lanes due to their strategic potential for the detection of passing vessels. Of particular interest is the development of wireless, acousticallylinked, total-field magnetic sensors with on-board detection and classification capabilities. As a result of this interest, in May 2009, Next Generation Autonomous Systems (NGAS) sea trials took place in the Bay of Taranto in southern Italy. During this trial, the wireless acoustically-linked helium-3 nuclear-precession total-field magnetometers and associated hardware discussed in [1], were tested.

In order to fully advance the detection and classification capabilites of the magnetic sensor based systems, it is imperative to understand the characteristics of the noise. In order to achieve this goal, a data set of ambient geomagnetic background noise was recorded during the NGAS 2009 trials. This data was then analyzed to determine the noise characteristics including: statistics, anomalies, diurnal patterns, and frequency power spectra.

Four categories of noise found in the data are presented: acoustic-modem induced noise, clock-drift induced noise, geomagnetic background fluctuations, and geomagnetic ULF pulsations. The acoustic-modem induced noise was removed with a low-pass filter, as discussed in [1], as it was outside the frequency band for signals of interest (SOI). The remaining three noise categories will be discussed here. Additionally, two effective noise suppression techniques are utilized - described in [2] for tri-axial magnetomers - including a gradiometer formed from a pair of magnetometers located a few hundred meters apart, and a linear prediction method to spectrally whiten the noise.

\section{NOISE DISCUSSION}

Four types of noise were analyzed from the NGAS data set. The noise can be subdivided into types as: modem induced noise, clock-drift induced noise, geomagnetic background noise, and geomagnetic ULF pulsation noise. The acousticmodem induced noise - eliminated by using a low-pass filter with a $30 \mathrm{mHz}$ cutoff frequency - was discussed in [1] and is not discussed here. The other three noise types are discussed next.

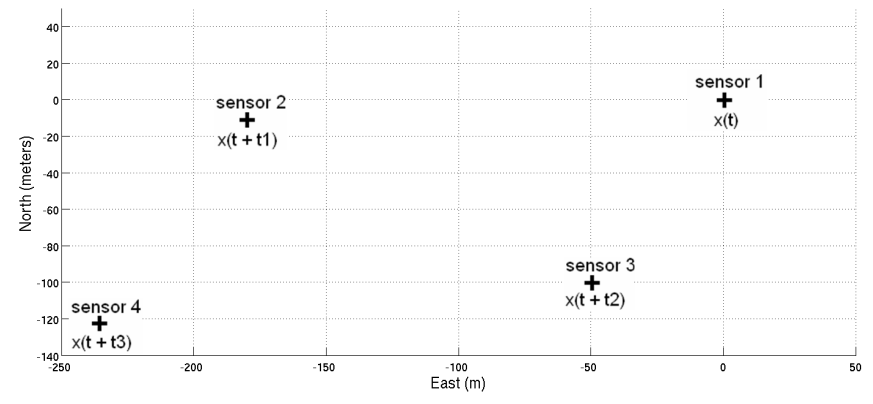

Fig. 1. Four-element sensor network with variable clock drift among sensors. 


\section{A. Clock-Drift Induced Noise}

A problem encountered during the NGAS sea trials was the presence of a variable sample offset between pairs of sensors due to internal sensor clock drift. To illustrate, consider Fig. 1, which depicts a deployed configuration of four sensors, such as that used by NGAS. Letting sensor 1 be the reference, the measurement $x(t)$ is offset in time for sensors 2,3 , and 4 , by $t_{1}, t_{2}$, and $t_{3}$ samples, respectively. Due to this sample offset, any time domain subtraction between sensors, or gradiometer measurements, would be compromised, including gradiometerbased noise suppression and target-detection collaboration.

Fortunately, the very property of the geomagnetic background noise that makes gradiometer-based noise suppression effective, can also be used to correct for clock drift. The geomagnetic background noise is spatially coherent at distances far exceeding that used in a magnetometer sensor network, such as that shown in Fig. 1. Therefore, assuming no strong local magnetic anomalies, such as a passing ocean vessel that will degrade coherence, are present in the recorded data, the cross-correlation [3] can be used to determine the sample offset between any pair of sensors. The cross-correlation is computed by:

$$
R(l)=\sum_{t=-\infty}^{\infty} x(t) y(t-l)
$$

where $l$ is the time shift or lag parameter, and the two sensor data sets are $x$ and $y$. The sample offset between sensor pairs is determined by computing $R(l)$ for all relevant values of $l$ and determining $l$ at which $R(l)$ is maximum. Fig. 2 depicts the cross-correlation computed between a pair of sensors and indicates the lag associated with the maximum value, which is the sample offset between the pair of sensors.

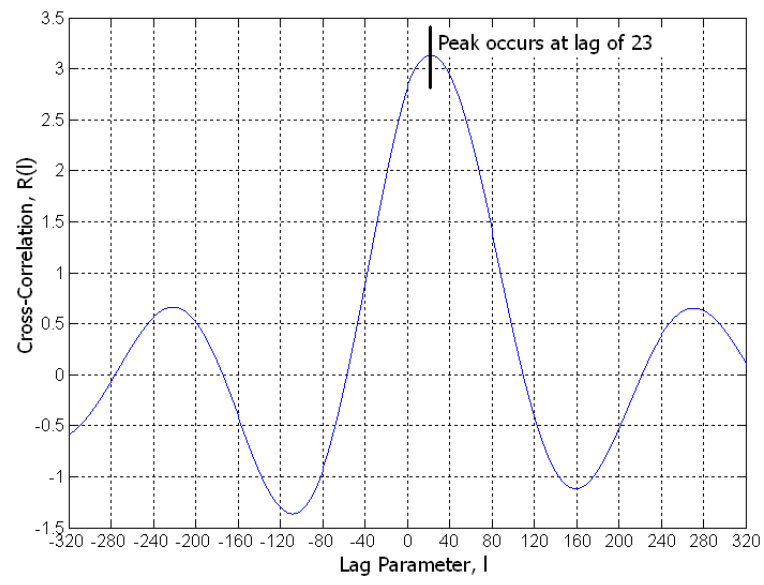

Fig. 2. Cross-correlation between a pair of sensors with sample offset indicated. Note that the peak of the cross-correlation indicates the estimated sample offset.

For the NGAS data set, the sample offset varied throughout the day. In order to quantify this variation, the sample offset between each sensor and a reference sensor was computed throughout the day in 23 minute segments (8000 samples at
TABLE I

SAMPLE OFFSET BETWEEN LISTED SENSORS AND SENSOR 1.

\begin{tabular}{|c||c||c||c|}
\hline Sample Lag & Sensor 2 & Sensor 3 & Sensor 4 \\
\hline Mean: & 22.5 & 35.7 & 26.9 \\
\hline Standard Deviation: & 2.7 & 2.7 & 2.5 \\
\hline Coefficient of Variation: & $11.8 \%$ & $7.6 \%$ & $9.1 \%$ \\
\hline
\end{tabular}

the $5.86 \mathrm{~Hz}$ sample rate). Table 1 presents the results of this analysis, where sensor 1 was used as the reference sensor. The mean sample offset between each listed sensor in Table 1, and sensor 1 was 23, 36, and 27 samples. If a gradiometer based noise suppression scheme was utilized, these sample offsets would superficially increase the noise floor by $20 \mathrm{~dB}$.

To illustrate the effect of not accommodating for clockdrift induced sample offsets, consider Fig. 3, which compares the power spectral density (PSD) of the gradometer in which the sensor pair was sample aligned, with the PSD of the non sample aligned gradiometer pair. In this case, the sample offset was 25 samples. From Fig. 3, it is seen that over a $20 \mathrm{~dB}$ loss in noise suppression occurs for the gradiometer that is not sample aligned.

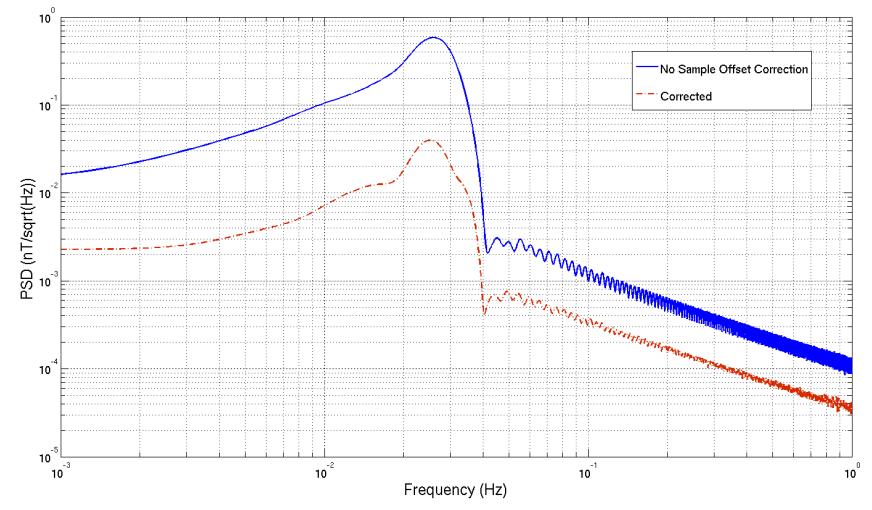

Fig. 3. PSD gradiometer comparison: sample offset corrected and sample offset uncorrected. A 25 sample alignment leads to an approximate $20 \mathrm{~dB}$ improvement in the PSD response.

Additionally, one must consider the time-varying nature of the clock drift. From Table 1, it is seen that clock drift varied by as much as $12 \%$ on a 23 minute interval. It was found that a sample offset between gradiometer sensor pairs of as little as 5 samples, could lead to a loss in noise suppression of $6 \mathrm{~dB}$. Considering that the static magnetic field component varies as the inverse of the range to the third power $\left(1 / R^{3}\right)$, small signals, on the order of a few nano-Tesla are common and can be suppressed by even 5 samples of drift.

In order to utilize multi-sensor information in a real-time system it is necessary to periodically recalculate the sample lag between deployed sensor pairs. However, this requires differentiation between incoherent events and coherent background geomagnetic fluctuations. If an incoherent event across the sensor field, such as an SOI, is mistakenly taken as coherent noise, an incorrect time alignment value will be calculated. 
Because clock drift values are periodically updated throughout the data collection, with expected drift on the order of a few samples over an hour long period, the drift values are updated and compared to previous values. If a value exceeds the previous calculation by more than an order of magnitude, it is identified as a section of data that had incoherent events and the previously calculated clock drift value is used.

\section{B. Geomagnetic Background Fluctuations}

By geomagnetic background fluctuations, we refer to those time-variations due primarily to small signal variations in the Earth's magnetic field, which are superimposed on the static magnetic field [4]. These fluctuations are spatially coherent at distances exceeding a few kilometers, typically far greater than that of the deployed magnetic sensors. Two effective methods of suppressing these background fluctuations include the use of a gradiometer, as well as linear prediction filtering [2]. Prior to presenting the effectiveness of these two noise suppression techniques on the NGAS data set, a brief description of the statistical properties of the NGAS data set is presented.

1) Statistical Properties: The diurnal and statistical properties of the band-pass filtered (BPF) (1 to $30 \mathrm{mHz}$ ) [1] background fluctuations are analyzed. The fluctuations are found to be non-stationary with variably-high kurtosis and skewness. Additionally, the variance reaches minimums around midnight of each day. Also, the spatial coherence between pairs of sensors is found to vary slightly throughout the day as well, presumably due to spurious man-made signals, which were not signals of interest. This variable spatial coherence, although slight, would reduce the effectiveness of gradiometer based noise suppression.

Fig. 4 plots the noise variance calculated in one hour segments throughout the NGAS data set. Hour 1 in Fig. 4 refers to 9 AM UTC. Around 1 AM UTC of each day, minimums in the variance occur, and at this time the variance is approximately $15 \mathrm{~dB}$ lower than at any other time throughout the day. During the remainder of the day, the variance fluctuates between +5 $\mathrm{dB}$ and $-5 \mathrm{~dB}$.

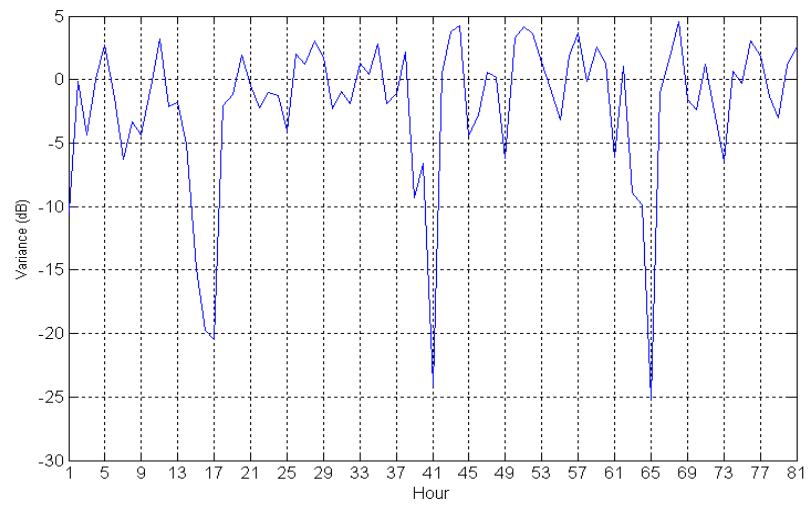

Fig. 4. Hourly noise variance, with minimum background noise occuring at $1 \mathrm{AM}$ on each of the three days.

The kurtosis and skewness, calculated in one hour segments throughout the data set, are presented in Fig. 5 and Fig. 6.
From Fig. 5, it is evident that the noise distribution is leptokurtic and the kurtosis varies throughout the day from between four and twenty. This indicates a very tight distribution around the mean. In Fig. 6, it can be seen that the noise distribution varies between positive and negative skew. Therefore, the noise distribution often has a relatively heavy tail that shifts between the left and right sides of the distribution.

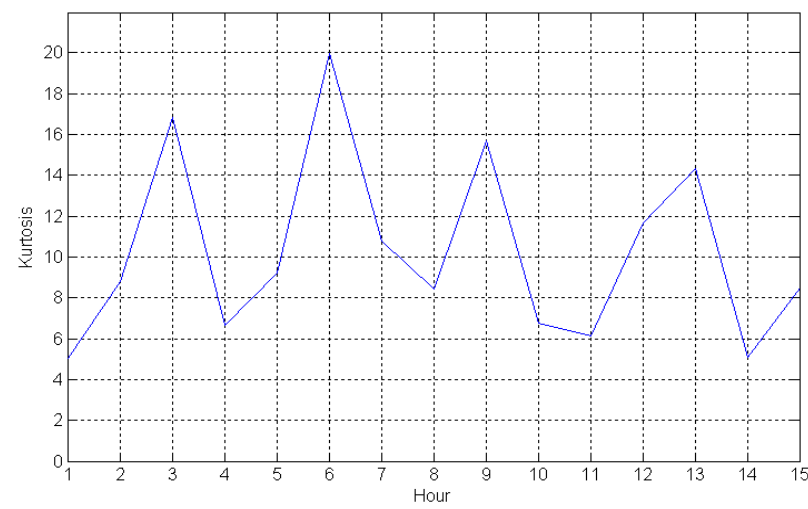

Fig. 5. Hourly noise kurtosis.

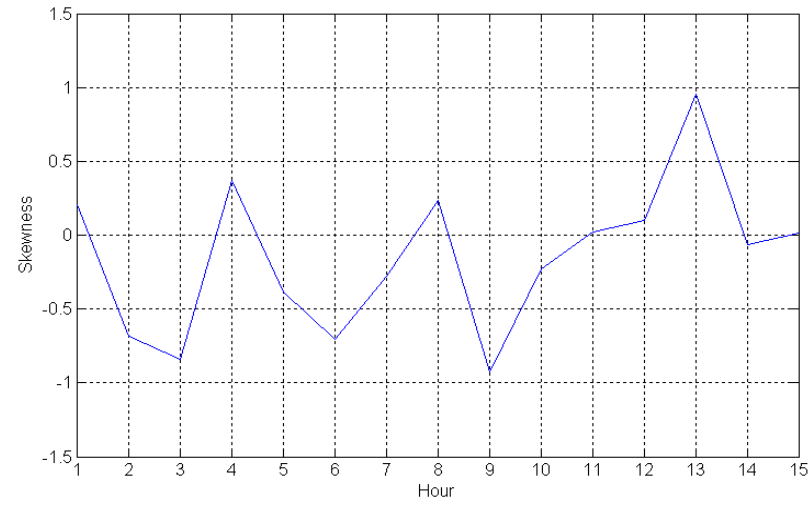

Fig. 6. Hourly noise skewness.

2) Noise Suppression Techniques: Two noise suppression techniques were utilized, including the use of a reference sensor to form a gradiometer, and a linear prediction or noise whitening filter. The results from using these techniques on the NGAS data set are presented and a comparison of each technique is made.

Fig. 7 provides a comparison between the PSD of a BPF NGAS data segment, the gradiometer of the BPF data segment, and the whitened version of the BPF data segment. For the noise whitening technique, a 20th order autoregressive was used to model the geomagnetic noise process. From Fig. 7 , it is evident that noise whitening proved superior to the gradiometer at reducing the in-band noise, with up to a $20 \mathrm{~dB}$ improvement in noise suppression. The whitening filter also provides a nearly flat spectral response in-band.

Although the whitening filter proved superior at noise suppression over the gradiometer, it is not without drawbacks. The whitening filter will create a spectrally flat region in the 


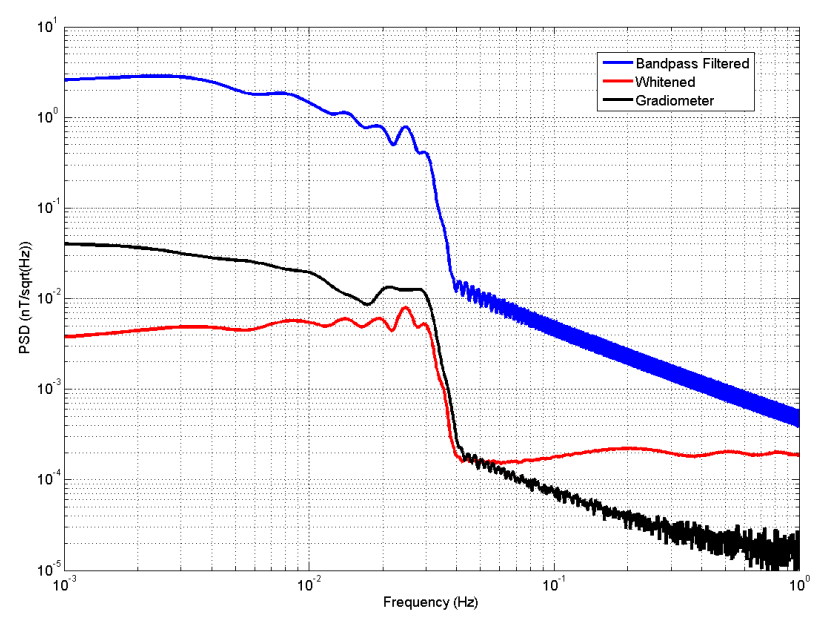

Fig. 7. Noise suppression comparison whitening vs. gradiometer.

entire pass band, including any SOI that may be present. More details regarding the whitening process can be found in [2].

Although the gradiometer was less effective than the whitening filter at noise suppression, it generally does not reduce the SOI. This is assuming the two sensors used to form the gradiometer are located sufficiently far apart from the passing SOI. However, the gradiometer method has several practical limitations for usage in a real-time system. The two sensors must be sample aligned so that spatially coherent samples will be reduced in magnitude when the gradiometer is computed. Therefore, this method first requires application of the method presented in Section II-A in order to time-align the data. Additionally, reference sensor data must be transmitted to other sensors, which requires a wired or wireless link. Given the impracticalities and constraints on deployment, seafloor cable footprint, and susceptibility to trawling, undersea wired links in a harbor-type environment are not being considered for this application. Additionally, data streaming over a low bandwidth underwater acoustic channel, utilizing data from spatially distributed sensors poses another set of challenges. Therefore, non gradiometer based methods, with no requirement for data exchange from distributed sensors should be explored.

The whitening technique presented requires the use of only a single sensor for noise suppression, requiring no data stream between sensor nodes. This makes it very desirable for usage in a real-time deployable system.

\section{ULF Pulsations}

ULF pulsations refer to intermittent magnetic signals, located in the ULF band, that are particularly strong relative to the more continuous geomagnetic background variations. Considering that the source of the ULF pulsastions is in the ionosphere, provided that the magnetic sensor distribution is on the order of a few hundred meters, these pulsations are coherent across the sensor field, just as other geomagnetic background fluctuations. Due to the irregular nature, and the fact that the pulsations are within our band of interest of 1 -
$30 \mathrm{mHz}$, it is believed that these pulsations could potentially be of the pi-2 variety [5], which are often classified as occurring between $7 \mathrm{mHz}$ and $22 \mathrm{mHz}$. However, they do not appear to follow the diurnal patterns associated with pi2 presented previously in the literature [6], which specifies a maximum occurrence of around midnight. Therefore, these pulsations could also be of the pc-4 variety, given their daytime occurrence and that pc-4 pulsations share the same frequency band as pi-2 pulsations. Regardless of their classification, these pulsations are problematic in a single sensor based detection scheme, consisting of noise whitening combined with matched filtering, as described in [1].

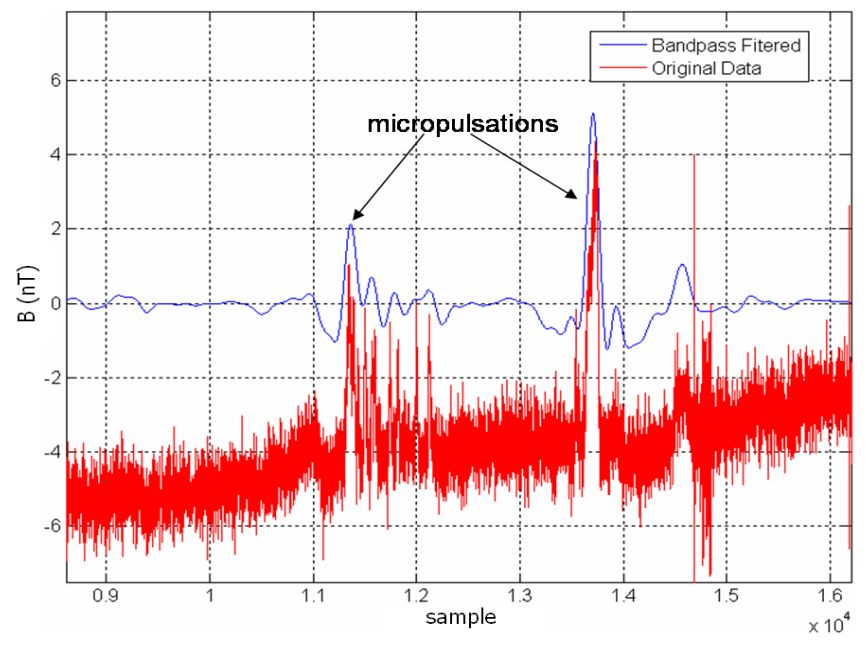

Fig. 8. Micropulsations: raw data and BPF data.

Fig. 8 provides an example of the ULF pulsations found within the NGAS data set. It presents both the unprocessed data and the BPF data. Note that the unprocessed data had its scale altered for presentation purposes. The pulsations are characterized by a sharp irregularity superimposed on the geomagnetic background fluctuations, that has a period between 45 to 150 seconds. The pulsations resemble an SOI, but, unlike an SOI, they are spatially coherent across the sensor field.

Fig. 9 illustrates the spatial coherence of the pulsations. It superimposes two sets of sensor data without sample-aligning them. From Fig. 9, it is clear that a gradiometer could be used to eliminate the pulsations. This spatial coherence is also an indication of a potential source for the pulsations. Given the coherence of the signals, the source is suspected to be on the order of tens to hundreds of kilometers distant from the magnetometers. Also, considering the $1 / R^{3}$ losses in signal strength, the source of the pulsations would need to be relatively strong. Therefore, it is believed that the source of the pulsations is in the atmosphere and not a local man-made phenomena.

Unfortunately, the ULF pulsations have characteristics dissimilar to that of the geomagnetic background fluctuations. Therefore, these pulsations are often relatively unaffected by noise whitening, or can cause whitening to reduce the presence 


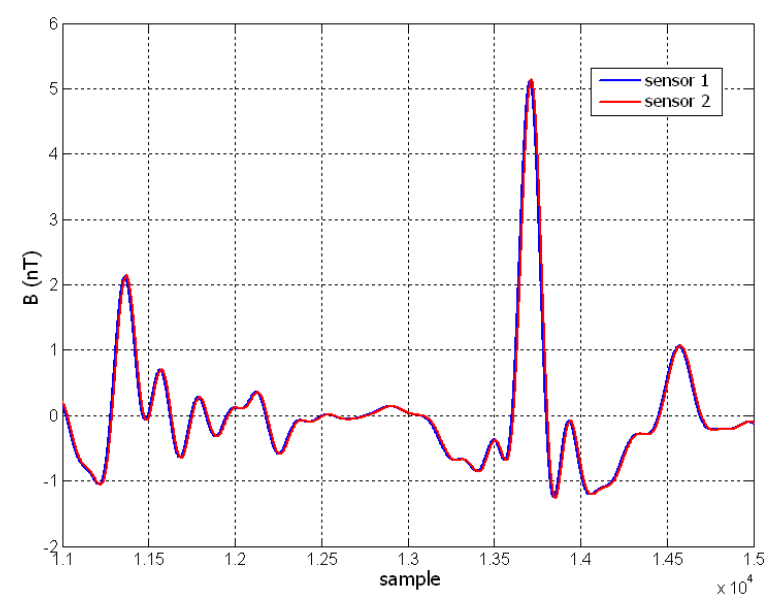

Fig. 9. ULF Pulsations: example of spatial coherence

of signals of interest. An example of this is provided using the data presented in Fig. 10. The synthetic target has a magnetic moment magnitude of $10^{5} \mathrm{Am}^{2}$, at a range of $200 \mathrm{~m}$, heading of $45^{\circ}$, and a speed of $4 \mathrm{~m} / \mathrm{s}$. Noise segments 1 and 2 represent the background noise used to generate the whitening filter coefficients that were used in generating Fig. 11 and Fig. 12, respectively. Note that noise segment 1 contains no ULF pulsations, whereas noise segment 2 does contain ULF pulsations.

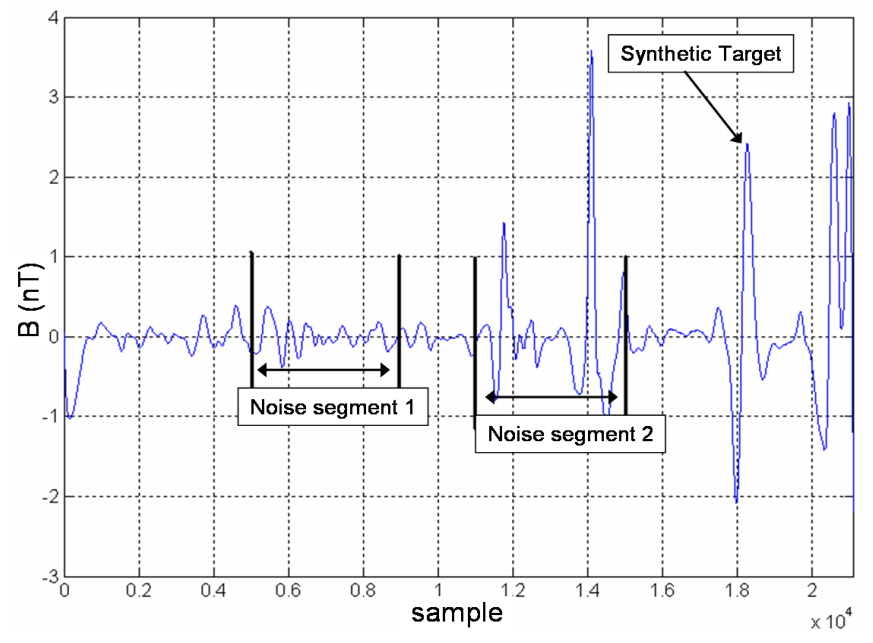

Fig. 10. Synthetic Target and Noise Segments used for Generating a Whitening Filter

Fig. 11 presents the results of whitening with filter coefficients obtained from noise segment 1 , combined with matched filtering [7] (labeled as MF in Fig. 11). The original BPF data, on which the whitening and matched filtering was performed, is also shown for comparison (labeled as BPF in Fig. 11). Notice that matched filtering provided a $0.8 \mathrm{~dB}$ improvement in SNR (16 dB for the matched filter data) over the BPF data SNR $(15.2 \mathrm{~dB})$. However, note that the ULF pulsations were not only unaffected by the whitening filter, but also had their signal strength increased by the matched filter.

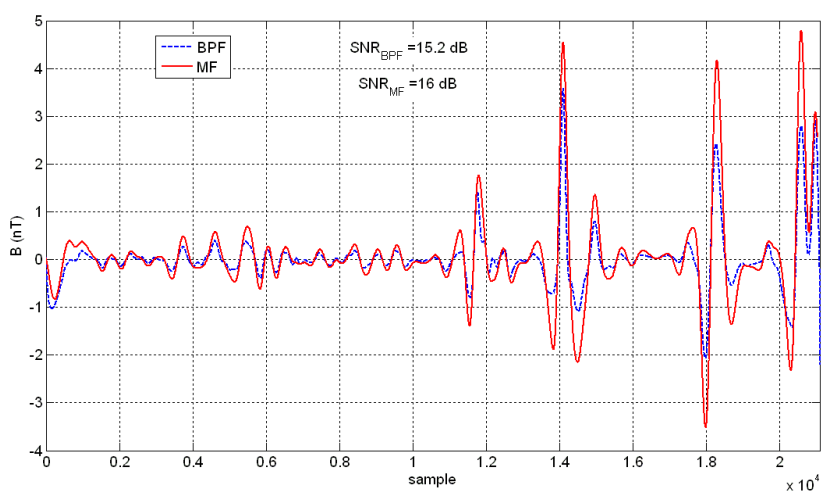

Fig. 11. Matched Filtering Combined with Whitening using Noise Segment 1 from Fig. 10

Next, the whitening filter coefficients were obtained from noise segment 2, and whitening and matched filtering were again performed on the bandpass filtered data, obtaining the result shown in Fig. 12. In this case, whitening combined with matched filtering provided a $0.9 \mathrm{~dB}$ loss in SNR relative to the bandpass filtered data. Also, it is evident that the whitening filter managed to predict the presence of the ULF pulsations and target signal, effectively reducing the signal strength of both.

The presence of ULF pulsations can significantly affect the capabilities of a single sensor detection system. They have the potential to both increase the false-alarm rate, and decrease the target-detection rate. A means of reducing or eliminating the negative effects of ULF pulsations needs to be further investigated.

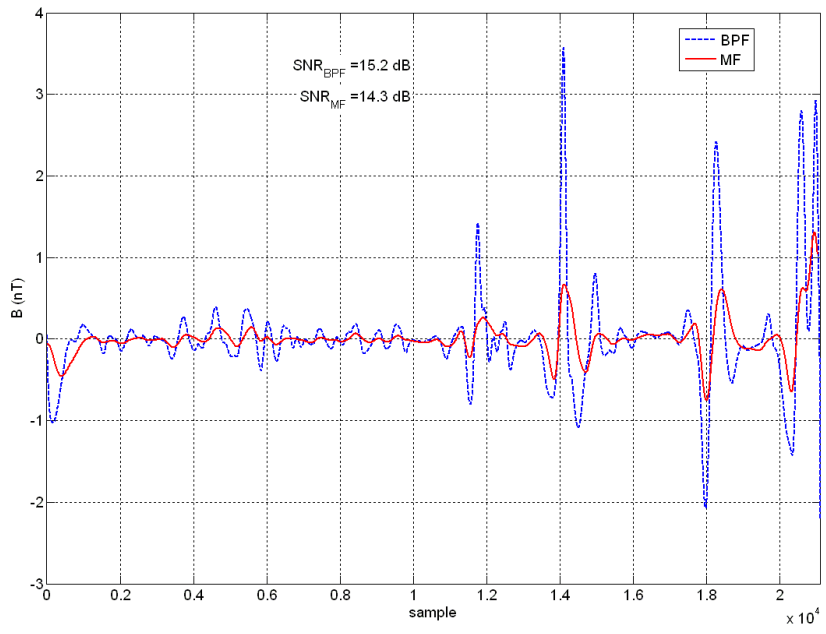

Fig. 12. Matched Filtering Combined with Whitening using Noise Segment 2 from Fig. 10

\section{CONCLUSION}

Geomagnetic noise data from the NGAS 2009 sea trial was analyzed. Four different types of noise found in the data were presented, including: acoustic-modem induced noise, clock- 
drift induced noise, geomagnetic background fluctuations, and geomagnetic ULF pulsations.

For the clock-drift induced noise, a method of using the cross-correlation of the geomagnetic background fluctuations obtained from a pair of sensors was presented for sample aligning the data. Due to the long-range spatial coherence of the geomagnetic noise, this technique proved quite effective. But, it is important that the data segment being used to compute the cross-correlation not contain any strong local magnetic signatures that would invalidate the spatial coherence assumption. Additionally, due to the time-varying nature of the clock drift, periodic updates of the sample lag between sensor pairs would be necessary. As an example, ignoring the sample lag was found to yield a $6 \mathrm{~dB}$ loss in noise suppression for a lag between sensor pairs of only 5 samples, which was within the normal daily variation.

The statistical and diurnal properties of the geomagnetic background fluctuations were then presented, along with results of noise suppression. The fluctuations were found to be non-stationary with variably-high kurtosis and skewness. Interestingly, the hourly variance fluctuated throughout the day, reaching minimums around $1 \mathrm{AM}$ that were $15 \mathrm{~dB}$ lower than the rest of the day.

Results from using the gradiometer and whitening filter noise suppression schemes were then presented. The whitening filter proved superior to the gradiometer at noise suppression within the band of interest by as much as $10 \mathrm{~dB}$. However, it was noted that the whitening filter also presents the possibility of weakening any SOI whereas the gradiometer (assuming sufficient sensor pair separation) does not.

Finally, geomagnetic ULF pulsations were presented. This noise type is spatially coherent, and is characterized by highamplitude irregular pulsations superimposed on the geomagnetic background noise. Although a gradiometer can easily eliminate the ill-effects of ULF pulsations, the pulsations can hinder single sensor detection schemes utilizing a whitening filter paired with a matched filter. Due to the resemblance of the ULF pulsations to signals of interest, the single sensor detector was found to either weaken both the pulsations and the SOI, or boost the signal strength of both.

Analysis of the NGAS data set will assist in the advancement of stationary, undersea, passive total-field magnetic sensor technologies, and associated signal processing algorithms. A pratical means of sample-aligning a sensor field with respect to each sensor, utilizing the presented cross-correlation method, will need to be implemented. Also, for a singlesensor detection scheme, a robust means of dealing with the geomagnetic ULF pulsations found throughout the NGAS data set will need to developed.

\section{ACKNOWLEDGMENT}

The authors would like to thank ONR 321 MS for their support of this work, in addition to the NGAS partners, including: DRDC Canada, FFI Norway, NURC, and the Italian Navy.

\section{REFERENCES}

[1] M. Tomic, P. T. Sullivan, and V. K. McDonald, "Wireless, acoustically linked, undersea, magnetometer sensor network," MTS/IEEE Oceans, Oct. 2009.

[2] R. Otnes, C. Lucas, and P. Holtham, "Noise suppression methods in underwater magnetic measurements," Proc. Marelec 2006, Apr. 2006.

[3] J. G. Proakis and D. G. Manolakis, Digital Signal Processing, 4th ed., Pearson Prentice Hall, Upper Saddle River, N.J., 2007, pp. 118-125.

[4] R. Craig, The Upper Atmosphere: Meteorology and Physics, Academic Press, Inc., New York, N.Y., 1965, pp. 362-379.

[5] M. G. Cardinal and K. Yumoto, "Characteristics of Equatorial Pc3 Pulsations," International Conference on Space Science and Communication, Oct. 2009.

[6] D. Orr and H. G. Barsczus "Geomagnetic Micropulsations, pi2, at Low Latitudes," Planetary and Space Science, Vol. 17, No. 3, Mar. 1969.

[7] R. Otnes, "Static magnetic dipole detection using vector linear prediction, Anderson functions, and block-based adaptive processing," IEEE Oceans Europe, pp. 1-6, Jun. 2007. 